\title{
Vascular Thrombosis and Anti-PD-I Therapy: A Series of Cases
}

\author{
Changfang Fu (D) \\ Gang Wang' \\ Wulin Yang ${ }^{2}$
}

IThe First Affiliated Hospital of USTC, Division of Life Sciences and Medicine, University of Science and Technology of China, Hefei, 23000I, People's Republic of China; ${ }^{2}$ Anhui Province Key Laboratory of Medical Physics and Technology, Institute of Health and Medical Technology, Hefei Institutes of Physical Science, Chinese Academy of Sciences, Hefei, 23003I, People's Republic of China
Correspondence: Wulin Yang Anhui Province Key Laboratory of Medical Physics and Technology, Institute of Health and Medical Technology, Hefei Institutes of Physical Science, Chinese Academy of Sciences, 350 Shushanhu Road, Hefei, 23003I, Anhui, People's

Republic of China

Email yangw@cmpt.ac.cn

Gang Wang

Oncology Department, The First Affiliated Hospital of USTC, Division of Life Sciences and Medicine, University of Science and Technology of China, No. 17 Lujiang Road, Luyang District, Hefei, 23000I, Anhui, People's Republic of China Email wanggang829@I26.com

\begin{abstract}
Immune checkpoint inhibitors may cause unique immune-related adverse events. Vascular thrombosis, especially arterial thrombosis, is rare but life-threatening, and little is known about its relevance to immunotherapy. Here, we reported two cases of vascular thrombosis, including venous and arterial thrombosis in cancer patients receiving anti-PD-1 antibody in combination with chemotherapy. Systemic corticosteroids and anticoagulant treatment were administered immediately in two cases. In case 1, anti-PD-1 antibody was permanently discontinued, and recurrence of vascular thrombosis was not observed during the follow-up. In case 2, the patient continued anti-PD-1 immunotherapy and unfortunately died of cerebral infarction 2 months later. This case report provides a strong evidence for the association between PD-1 blockade and vascular thrombosis and offers some general guidelines on the management of the immune-related vascular thrombosis events induced by anti-PD-1 therapy.
\end{abstract}

Keywords: immune checkpoint inhibitors, immune-related adverse events, pembrolizumab, tislelizumab, pulmonary embolism, deep vein thrombosis

\section{Introduction}

Immune checkpoint blockade may cause unique immune-related adverse events (irAE). Vascular thrombosis, including venous and arterial thrombosis, is rarely known to be related to immunotherapies. ${ }^{1,2}$ It has been recently reported that the cumulative incidences of venous thrombosis and arterial thrombosis were $12.9 \%$ and $1.8 \%$ in a cohort of patients treated with immune checkpoint inhibitors, respectively. ${ }^{3}$ Patients with cancer immunotherapy such as immune checkpoint blockade are at high risk of thromboembolism. ${ }^{1,4}$ Drug withdrawal, early engagement with systemic corticosteroids and anticoagulant treatment might be important for preventing vascular thrombosis after immunotherapy. ${ }^{5}$ More insight into the thromboembolism associated with immunotherapies is warranted to identify the clinical management for thromboembolism.

Pembrolizumab and tislelizumab, humanized IgG4 anti-programmed cell death1 (PD-1) monoclonal antibody specifically designed to bind to PD-1 to restore the ability of cytotoxic T-lymphocytes to kill cancer, are being developed as a monotherapy and in combination with chemotherapy for the treatment of a broad array of both solid and hematologic cancers. Here, we report two cases of vascular thrombosis in cancer patients receiving anti-PD-1 antibody in combination with chemotherapy. This work provides some general guidelines on the management of these immune-related vascular thrombosis events. 


\section{Case I}

A 66-year-old woman with a history of hypertension and cerebral infarction was readmitted with chest tightness and shortness of breath. One month ago, she come to our hospital with a 2-month history of progressive headache. Magnetic resonance imaging (MRI) of the brain demonstrated an abnormal signal in the right occipital bone and adjacent intracranial soft tissue. Gastroscopy biopsy revealed deep ulcerative lesions with gastric adenocarcinoma cells. A computed tomography (CT) scan of the chest was performed and demonstrated a mass in the left lung and multiple nodules in bilateral lungs. Therefore, she was diagnosed with gastric cancer of clinical-stage IV with lung and brain metastasis and treated with the combination tislelizumab 200mg day 1 and chemotherapy of oxaliplatin $130 \mathrm{mg} / \mathrm{m}^{2}$ day 1 and capecitabine $1000 \mathrm{mg} / \mathrm{m}^{2}$ bid day $1-14$ every 3 weeks.

The patient complained of cough, phlegm and expectoration after the initial administration. Auscultation revealed diminished breath sounds in the bilateral lower lung. Edema was found in both lower extremities. Laboratory test revealed serum C-reactive protein (CRP) and D-dimer levels elevated. Pulmonary embolism (PE) was suspected and emergency CT pulmonary angiography (CTPA) and Doppler ultrasound were undergoing. CTPA revealed acute bilateral PE and bilateral pleural effusions (Figure 1A). Doppler ultrasound examination detected evidence of deep vein thrombosis (DVT) in the right posterior tibial vein (Figure 1B).

The DVT with PE and pleural effusion were considered to be irAE induced by tislelizumab based on the criteria for assessing adverse drug reactions after discussion between clinicians and clinical pharmacists. Tislelizumab was permanently discontinued. Systemic corticosteroids with methylprednisolone $40 \mathrm{mg}$ and anticoagulant treatment with low-molecular-weight heparin (LMWH) 3200 units were administered twice a day immediately. Thoracentesis was performed to relieve the compression symptoms of pleural effusion. Clinical improvement was noted, so methylprednisolone was reduced to $40 \mathrm{mg}$ once daily and then gradually reduced until complete cessation. After discharge, the patient was given long-term oral anticoagulation with rivaroxaban $15 \mathrm{mg}$ bid day $1-20$ then $20 \mathrm{mg}$ qd after day 21 . No recurrence of thromboembolism was observed during 1-2 months of follow-up.

\section{Case 2}

A 70-year-old man was diagnosed with metastatic cholangiocarcinoma and treated with a combination of pembrolizumab, gemcitabine and cisplatinum every 3 weeks in a double-blinded study, where the dosage of pembrolizumab were $2 \mathrm{mg} / \mathrm{kg}$, the dosage of gemcitabine were $1000 \mathrm{mg} / \mathrm{m}^{2}$ and the dosage of cisplatinum were $25 \mathrm{mg} / \mathrm{m}^{2}$. After three injections, two months after the initial treatment, a routine CT scan revealed an arterial thrombus floating in the left pulmonary artery and branches with a bilateral femoral vein thrombosis (Figure 2A and B). D-dimer levels elevated.

Anticoagulant treatment with LMWH 4000 units was administered twice a day immediately. The patient had no obvious symptoms including cough, chest tightness, panting, shortness of breath, dyspnoea, pain and swelling of the lower extremities. Regular and systematic assessments of tumor burden showed the patient had stable disease. DVT combined with PE was initially thought to be
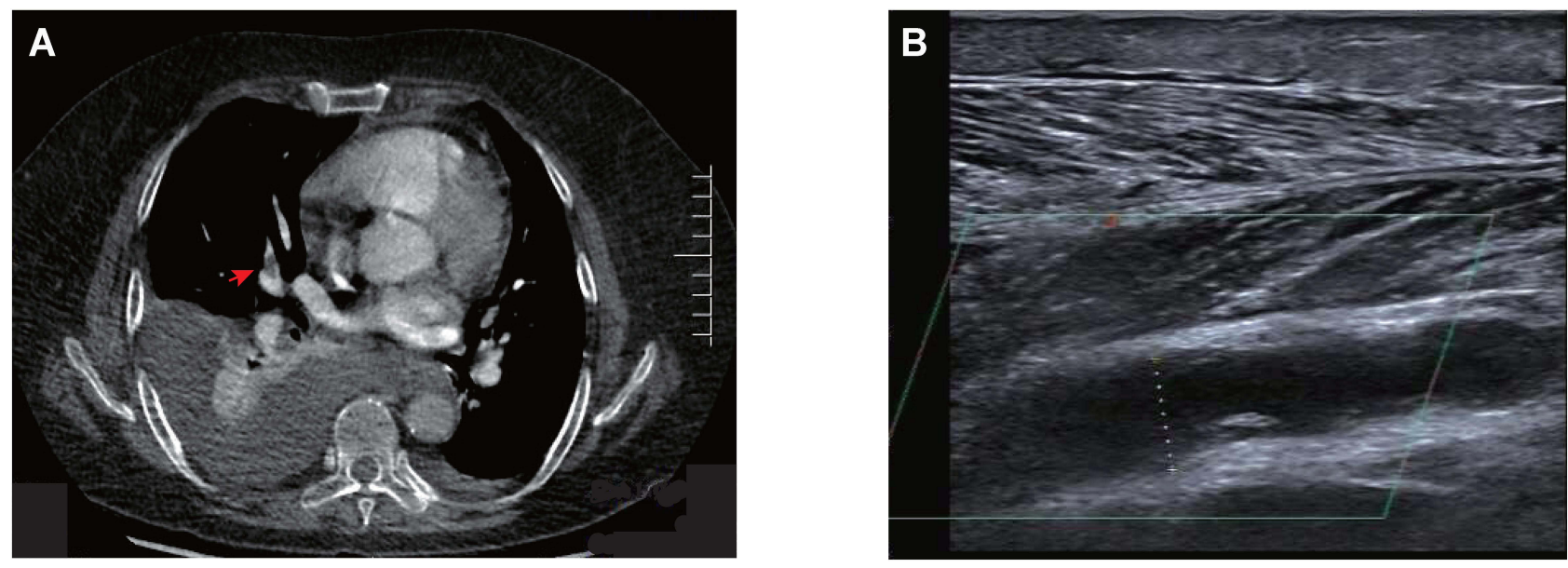

Figure I (A) Computed tomography pulmonary angiography (CTPA) revealed acute bilateral PE, bilateral pleural effusion with partial dilatation of the lower lobes and multiple discrete pulmonary nodules. (B) Doppler ultrasound examination detected evidence of deep vein thrombosis (DVT) in the right posterior tibial vein. The right posterior tibial vein was dilated, about $10.6 \mathrm{~mm}$ at the deepest point, with hypoechoic filling. 

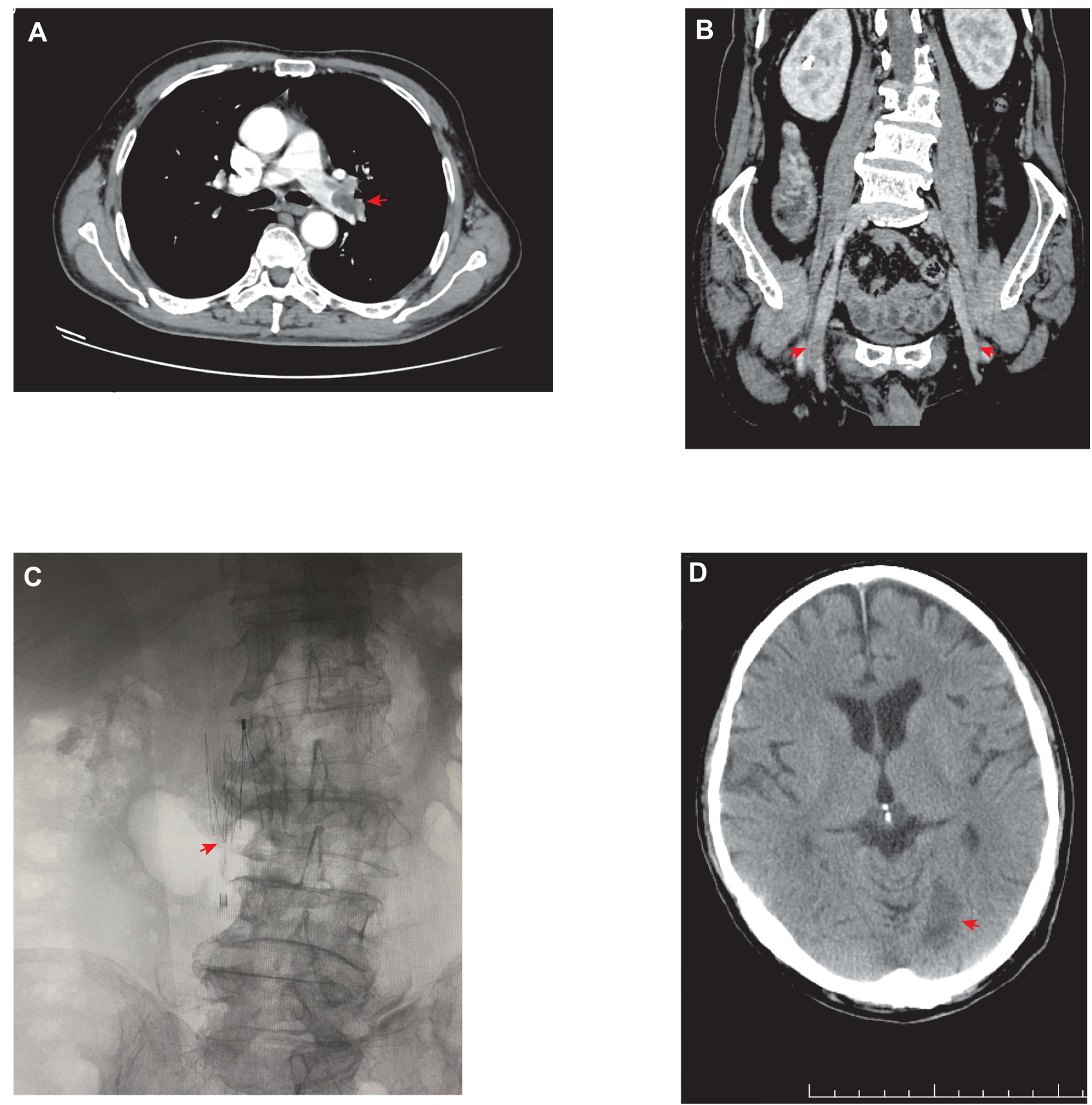

Figure 2 Computed tomography scan revealed a thrombus floating in the left pulmonary artery and branches (A) with a bilateral femoral vein thrombosis (B). Digital subtraction angiography (DSA) showed inferior vena caval filters were implanted in the inferior vena at L2 level with luminal patency (C). Emergency computed tomography (CT) scan showed cerebral infarction (D).

unrelated to anti-PD-1 therapy due to the poorly understood association between thromboembolism and cancer immunotherapy. Considering that the patient's clinical manifestation of thromboembolism was not obvious, and that the patient had shown an effective response to immunotherapy, and that the patient himself had requested continued immunotherapy, the multidisciplinary team (MDT) suggested the patient continued on the same therapy of a combination of pembrolizumab, gemcitabine and cisplatinum. In the meantime, oral anticoagulation with rivaroxaban was given and inferior vena caval filters were implanted for preventing fatal PE (Figure 2C). The patient was given long-term oral anticoagulation with rivaroxaban $15 \mathrm{mg}$ bid day $1-20$ then $20 \mathrm{mg}$ qd after day 21 . Two months later, after receiving another 3 doses, the patient visited the emergency department for cerebral infarction 
(Figure 2D). The patient develops hemiplegia, coma, loss of consciousness, and eventually death.

\section{Discussion}

It is well-known that cancer is related to vascular thrombosis due to multiple risk factors and complex mechanisms. Cancer patients are at increased risk for vascular thrombosis. Vascular thrombosis, particularly arterial thrombosis is rare but life-threatening and rarely known to be related to immunotherapies. ${ }^{6}$ Immune checkpoint blockade such as anti-PD-1 or its ligand PD-L1 as well as anti-cytotoxic $\mathrm{T}$ lymphocyte-associated antigen 4 (CTLA-4) monoclonal antibodies is the most promising treatment approach for cancers. It has been reported that IrAE is mainly related to the activation of the immune system during the anti-PD-1 treatment and is commonly observed in the skin, gastrointestinal tract, liver and endocrine systems. Previous studies strongly suggested that PD-1 blockade may induce accelerated inflammation which may cause thrombosis. ${ }^{7}$ Several studies suggest PD-1 can down-regulate $\mathrm{T}$ cell responses and PD-1 blockade may increase the risk of cardiovascular complications. ${ }^{8}$ A preclinical study confirmed the important role of PD-1 pathway in vascular inflammatory diseases. ${ }^{5}$ The interaction between macrophage and malignant cells could damage the endothelium, promote platelet deposition and thrombus growth. ${ }^{9}$ Anti-PD-1 antibody may also trigger a systemic immune response and IrAE. In this study, we report two cancer patients suffering from venous and arterial thrombosis induced by the anti-PD-1 antibody. After PE and DVT were confirmed, systemic corticosteroids and anticoagulant treatment were administered immediately in two patients. In case 1, tislelizumab was permanently discontinued and no recurrence of vascular thrombosis was observed. In case 2, the patient continued on the pembrolizumab in combination and died of cerebral infarction.

No definitive association has been established between venous and arterial thrombosis and anti-PD-1 therapy, so its risk has been underestimated in clinical practice. Once diagnosed, early anticoagulant treatment should be effective. Moreover, drug withdrawal and corticosteroids are typically the first line of treatment for most high-grade irAEs. Considering the threat of thrombosis to life, the clinical application of anti-PD-1 therapy must be vigilant. Given this, we report the occurrence of venous and arterial thrombosis associated with anti-PD-1 antibody therapy and develop a response plan to optimize the use of an antiPD-1 antibody. This case report provides further evidence of the association between PD-1 blockade and vascular thrombosis. We recommend that immediate drug withdrawal, anticoagulant and systemic corticosteroids can improve important clinical outcomes. Further studies must be conducted in the future to develop guidelines for prevention and response to vascular thrombosis induced by immune checkpoint blockade.

\section{Ethics Approval}

The study was approved by the ethics committee of the First Affiliated Hospital of USTC, Division of Life Sciences and Medicine, University of Science and Technology of China.

\section{Consent for Publication}

Written informed consents were obtained from the patients for publication of two cases report and any accompanying images.

\section{Funding}

No funding was available for this study.

\section{Disclosure}

The authors report no conflicts of interest in this work.

\section{References}

1. Roopkumar J, Swaidani S, Kim AS, et al. Increased incidence of venous thromboembolism with cancer immunotherapy. Med. 2021;2 (4):423-434. doi:10.1016/j.medj.2021.02.002

2. Iba T, Levy JH. Inflammation and thrombosis: roles of neutrophils, platelets and endothelial cells and their interactions in thrombus formation during sepsis. $J$ Thromb Haemost. 2018;16(2):231-241. doi:10.1111/jth.13911

3. Moik F, Chan WE, Wiedemann S, et al. Incidence, risk factors, and outcomes of venous and arterial thromboembolism in immune checkpoint inhibitor therapy. Blood. 2021;137(12):1669-1678. doi:10.1182/ blood.2020007878

4. Tsukamoto J, Monteiro M, Vale S, et al. Thromboembolic events related to treatment with checkpoint inhibitors: report of two cases. Case Rep Oncol. 2018;11(3):648-653. doi:10.1159/000492463

5. Boutros C, Scoazec J-Y, Mateus C, Routier E, Roy S, Robert C. Arterial thrombosis and anti-PD-1 blockade. Eur $J$ Cancer. 2018;91:164-166. doi:10.1016/j.ejca.2017.11.018

6. Di Nisio M, van Es N, Buller HR. Deep vein thrombosis and pulmonary embolism. Lancet. 2016;388(10063):3060-3073. doi:10.1016/ S0140-6736(16)30514-1

7. Bu DX, Tarrio M, Maganto-Garcia E, et al. Impairment of the programmed cell death-1 pathway increases atherosclerotic lesion development and inflammation. Arterioscler Thromb Vasc Biol. 2011;31 (5):1100-1107. doi:10.1161/ATVBAHA.111.224709

8. Kunimasa K, Nishino K, Kimura M, et al. Pembrolizumab-induced acute thrombosis: a case report. Medicine. 2018;97(20):e10772. doi:10.1097/MD.0000000000010772

9. Luther N, Shahneh F, Brahler M, et al. Innate effector-memory T-cell activation regulates post-thrombotic vein wall inflammation and thrombus resolution. Circ Res. 2016;119(12):1286-1295. doi:10.11 61/CIRCRESAHA.116.309301 


\section{Publish your work in this journal}

Cancer Management and Research is an international, peer-reviewed open access journal focusing on cancer research and the optimal use of preventative and integrated treatment interventions to achieve improved outcomes, enhanced survival and quality of life for the cancer patient.
The manuscript management system is completely online and includes a very quick and fair peer-review system, which is all easy to use. Visit http://www.dovepress.com/testimonials.php to read real quotes from published authors. 